\title{
ENERGETIC MATERIALS AT HIGH COMPRESSION: FIRST- PRINCIPLES DENSITY FUNCTIONAL THEORY AND REACTIVE FORCE FIELD STUDIES
}

\author{
I.I. Oleynik ${ }^{1}$, M. Conroy ${ }^{1}$, S.V. Zybin ${ }^{2}$, L. Zhang ${ }^{2}$, A.C. van Duin ${ }^{2}$, \\ W.A. Goddard III ${ }^{2}$ and C. T. White ${ }^{3}$ \\ ${ }^{1}$ Department of Physics, University of South Florida, Tampa, FL 33620 \\ ${ }^{2}$ Materials and Process Simulation Center, California Institute of Technology, Pasadena, CA 91125 \\ ${ }^{3}$ Naval Research Laboratory, Washington, DC 20375
}

\begin{abstract}
We report the results of a comparative study of pentaerythritol tetranitrate (PETN) at high compression using classical reactive interatomic potential ReaxFF and first-principles density functional theory (DFT). Lattice parameters of PETN I, the ground state structure at ambient conditions, is obtained by ReaxFF and two different density functional methods (plane wave and LCAO pseudopotential methods) and compared with experiment. Calculated energetics and isothermal equation of state (EOS) upon hydrostatic compression obtained by DFT and ReaxFF are both in good agreement with available experimental data. Our calculations of the hydrostatic EOS at zero temperature are extended to high pressures up to $50 \mathrm{GPa}$. The anisotropic characteristics of PETN upon uniaxial compression were also calculated by both ReaxFF and DFT.
\end{abstract}

Keywords: Energetic materials, PETN, interatomic potentials, molecular dynamics, EOS

PACS: $62.50 .+\mathrm{p}$, 82.40.Fp, 81.30.Hd, 46.40.Cd

\section{INTRODUCTION}

Prediction of properties of energetic materials using atomic-scale simulations techniques is one of the challenging areas of energetic materials (EM) research. Molecular dynamics (MD) simulation of EM using classical reactive interatomic potentials is a powerful modeling technique that is capable of addressing sub-nanometer and sub-picosecond length and time scales of shock compression and detonation phenomena. However, the results of computer simulations can only be as reliable as the ability of the interatomic potentials to properly describe a variety of chemical effects including bond-breaking and bond-making.
Recently, the reactive force field ReaxFF has been developed based on fitting of an ab-initio database of H-C-N-O chemistry and is currently being actively used for MD simulations of EM [13]. One of the important issues is the transferability of ReaxFF, i.e. its ability to describe a rich chemistry and physics of energetic materials compressed at high pressures and temperatures. The reliable experimental data mostly exist only for static volumetric compressions that produce the isothermal equation of states (EOS) in a limited range of pressures and temperatures. Although this information is useful to validate reactive interatomic potentials, it is very limited in nature. Additional atomic-scale information is urgently needed to understand the fundamental mechanisms 
of shock compression and detonation of EM materials. The modeling/simulation is able to provide such information that is very difficult or sometimes impossible to obtain from experiment.

Density functional theory (DFT) has been very successful in recent years in simulating and predicting properties of a wide spectrum of materials from first-principles, i.e. without ad-hoc parameters that are usually present in empirical and semi-empirical methods. In many cases the accuracy of DFT in simulating properties of condensed phases is within a few percent compared to experiment. However, systems with weak van der Waals interactions, such as energetic molecular crystals, comprise a real challenge for DFT, because various density functionals, including the local density approximation (LDA) and the generalized gradient approximation (GGA) face difficulties in describing systems having very small overlap of electronic densities from atoms constituting the system. Nevertheless, the DFT should work reasonably well for energetic materials at high pressures (because of substantial overlap of electronic densities from atoms) and can be a very efficient tool for generating a database of first-principles data that can be used for validation and fitting of interatomic potentials.

The purpose of this work is to perform a comparative study of static high pressure properties of pentaerythritol tetranitrate PETN using both density functional theory (DFT) and ReaxFF. PETN is one of the classical energetic materials that has been extensively studied in recent years

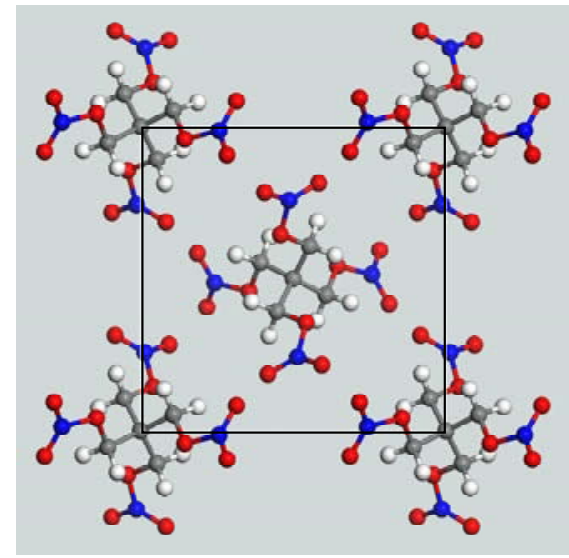

FIGURE 1. Crystal structure of PETN-I (space group symmetry $\mathrm{P} \overline{42}{ }_{1} \mathrm{C}$ ).
[4]. In addition to being an important secondary explosive, PETN exhibits an interesting property of strong anisotropy in the response to shock initiation of detonation [4]. Therefore, we decided to use PETN as a test bed for comparative studies using ReaxFF and DFT. We are particularly interested in equilibrium properties of the PETN stable crystalline phase at ambient conditions (PETN-I), see FIGURE 1, and its equation of state (EOS) in a wide range of applied pressures, see FIGURE 2.

\section{COMPUTATIONAL DETAILS}

The reactive force field (ReaxFF) is a bond-order dependent interatomic potential that includes covalent interactions via bond-orders. The bondorder is calculated from the instantaneous interatomic distances that are continuously updated in the course of simulation, thus allowing creation and breaking of chemical bonds. In addition to covalent interactions, ReaxFF includes Coulomb and van der Waals interactions traditionally present in classical force fields. However, these terms that are calculated for each atomic pair are effectively screened at short interatomic distances, thus reducing their contribution in the region where covalent interactions dominate. The ReaxFF was fitted to an extensive database of molecular and crystal structures of C-H-N-O containing species including reaction pathways, crystal structures and binding energy curves for all possible bonds including bond angle and torsional angle dependences [1-3]. Recently, ReaxFF has been successfully applied to study thermal decomposition of nitromethane [2] and RDX [3].

Density functional calculations were performed by using to different basis sets: plane waves (PW) and linear combination of atomic orbitals (LCAO). In both cases, high quality pseudopotentials were employed to remove the core electrons from calculations, thus making first-principles calculations feasible. We carefully studied the completeness of the plane wave basis set by optimizing energy cutoff to get the convergence in energies, forces and stresses better than 0.01

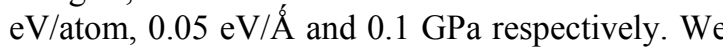
used Vanderbilt ultrasoft pseudopotentials (USPP) with $500 \mathrm{eV}$ energy cutoff, additional calculations were performed with $700 \mathrm{eV}$ to check the 
Table 1. Equilibrium lattice constants of PETN at zero temperature calculated by ReaxFF and DFT codes Castep and SeqQuest using several density functionals (LDA, GGA-PW, and GGA-PBE) and compared with experiment.

\begin{tabular}{|l|c|c|c|c|}
\hline \multicolumn{1}{|c|}{ PETN-I } & $\mathrm{a}=\mathrm{b}, \AA$ (error, \%) & $\mathrm{c}, \AA$ (error, \%) & $\mathrm{c} / \mathrm{a}$ (error, \%) & Cell Volume, $\AA$ \\
\hline Experiment & 9.38 & 6.71 & 0.71 & 589.5 \\
\hline LDA (Castep) & $8.891(-5.2 \%)$ & $6.453(-3.8 \%)$ & $0.726(+2.3 \%)$ & $510.1(-13.5 \%)$ \\
\hline GGA-PW (Castep) & $9.600(+2.3 \%)$ & $6.796(+1.3 \%)$ & $0.708(-0.3 \%)$ & $626.3(+6.2 \%)$ \\
\hline GGA-PBE (Castep) & $9.820(+4.7 \%)$ & $6.950(+3.6 \%)$ & $0.708(-0.3 \%)$ & $670.2(+13.7 \%)$ \\
\hline GGA-PBE (SeqQuest) & $9.702(+3.4 \%)$ & $6.889(+2.7 \%)$ & $0.710(0.0 \%)$ & $648.5(+10.0 \%)$ \\
\hline ReaxFF & $9.427(+0.5 \%)$ & $6.989(+4.2 \%)$ & $0.741(+4.4 \%)$ & $621.1(+5.4 \%)$ \\
\hline
\end{tabular}

convergence. The Brillouin k-point sampling with k-point density of $0.08 \AA^{-1}$ was chosen to get the energies, forces and stresses converged to values cited above. We used the plane wave code Castep [5].

LCAO DFT calculations have been performed using LCAO code SeqQuest that uses normconserving pseudopotentials (NCPP) and highquality contracted Gaussian basis sets [6]. It is a computationally efficient code that enables very large-scale calculations using rather modest computational resources. For present the calculations, the basis sets of double-zeta plus polarization (DZP) quality have been used with kpoint sampling of the Brillouin zone with similar density as used in plane-wave calculations.

\section{RESULTS AND DISCUSSION}

The critical issue in employing DFT methods to study molecular crystals is the choice of the appropriate density functional. We investigated the accuracy of several density functionals including standard local density approximation (LDA) and several generalized gradient approximation (GGA) functionals including Perdew-Wang (PW) and Perdew-Burke-Ernzerhof (PBE) by calculating the equilibrium lattice parameters of PETN-I. Table 1 compares the results of DFT calculations using ReaxFF, CASTEP and PW-USPP and LCAO codes with experiment. As expected, the LDA gives strong overbinding of weak van der Waals forces which results in lattice parameters 5\% smaller than experimental values. The best results were obtained using GGA-PW in case of PW-
USPP (errors less than 2.3\%) and GGA-PBE (errors less than 3.4\%) for LCAO-NCPP. ReaxFF also predicts lattice constants in good comparison with experiment.

The hydrostatic equation of states (EOS) for PETN-I was obtained by performing constant pressure calculations at zero temperature. Castep data were generated using variable cell optimization under the constraint of a diagonal stress tensor with fixed values of diagonal matrix elements equal to a desirable pressure. The ReaxFF calculations were performed using damped constant pressure molecular dynamics which is equivalent to conjugate gradient minimization of the total energy. This method also allowed the optimization of the unit cell of the crystal under the constant pressure constraint. The space symmetry of the crystal structure has been removed in order to relax symmetry imposed constraints. The atomic coordinates were also simultaneously optimized to get zero forces on atoms.

Isothermal EOS, i.e. the dependence of pressure on volume, for PETN-I obtained from the constant pressure simulations by Castep and ReaxFF are shown in FIGURE 2 and compared with experimental data by Olinger et al [7]. Both Castep and ReaxFF data compare well with each other as well as with experiment. The pressure range covered by our simulations extends beyond the experimental pressures up to $50 \mathrm{GPa}$. The energy changes in $\mathrm{eV} /$ atom relative to the equilibrium zero pressure structure of PETN-I are also predicted by ReaxFF in good agreement with DFT results.

We also performed an investigation of uniaxial compression of PETN. It is well-known that PETN shows interesting sensitivity properties, i.e. strong 

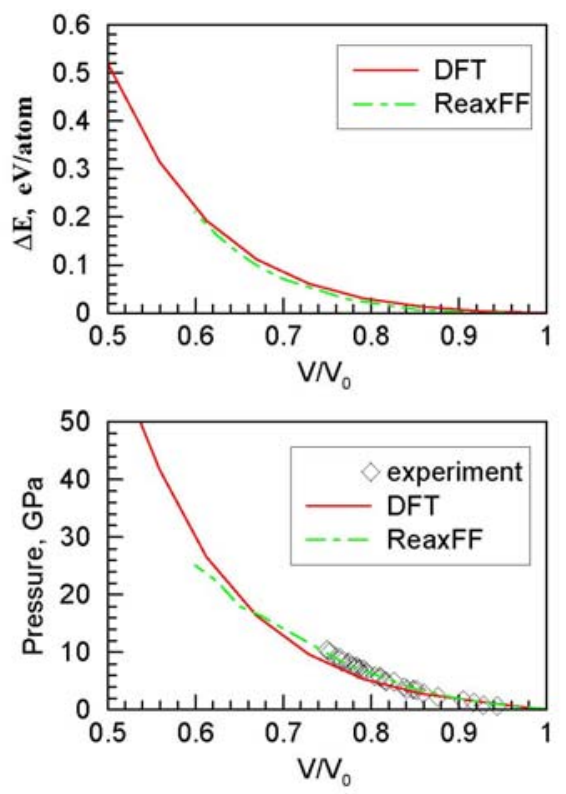

FIGURE 2. Constant pressure calculations of EOS for PETN-I. Top panel: energy changes relative to the equilibrium zero pressure structure of PETN-I. Bottom panel: the isothermal EOS of PETN-I.

anisotropy in the response in shock initiation of detonation. The shock loading conditions are characterized by fast uniaxial compression of the crystals along specific crystallographic directions. Therefore, we decided to investigate the predictions of both DFT and ReaxFF for uniaxial loading of the PETN-I.

The calculations were performed by creating a supercell where the PETN crystal is rotated to be oriented along a particular direction coinciding with $\mathrm{x}$-axis of the unit cell, resulting in a nontetragonal unit cell. Then, the $\mathrm{x}$-dimension of the unit cell was strained by an appropriate scaling of the lattice parameter $a$. We calculated the characteristics of uniaxially-loaded PETN-I crystal in [100], [110], [010], and [001] directions. Due to space limitations we show only [100] results in Figure 3. We found that the DFT-SeqQuest and ReaxFF are in very good agreement and it is not surprising, because ReaxFF was fitted using a database generated by SeqQuest. However, Castep calculations substantially deviate from both ReaxFF and SeqQuest. One of the sources of this disagreement is different GGA functionals: Castep calculations were done with PW but SeqQuest -

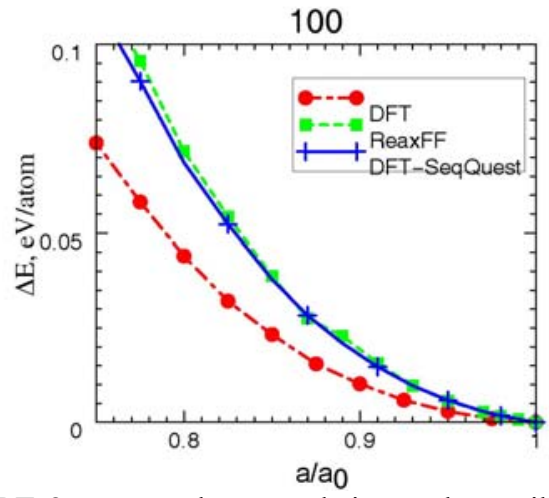

FIGURE 3. Energy changes relative to the equilibrium zero-pressure structure of PETN-I upon uniaxial compression in [100] direction.

with PBE functionals. This results in slightly different lattice constants for the equilibrium crystal structures of PETN, see TABLE 1, which may affect the curvature of the $\Delta E\left(a / a_{0}\right)$ curve. We are currently investigating the sources of this disagreement and will report the full results in a subsequent publication.

\section{ACKNOWLEDGEMENTS}

IIO is supported by NSF-NIRT (ECS-0404137) and ARO-MURI (W901 1NF-05-1-0266). Funding at Caltech was provided by ONR and ARO-MURI. CTW is supported by ONR directly and through Naval Research Laboratory.

\section{REFERENCES}

1. van Duin A.C.T., Dasgupta S., Lorant F. and Goddard III W.A., J. Phys. Chem. A 105, 9396 (2001).

2. Strachan A. et al, Phys. Rev. Lett. 91, 098301 (2003).

3. Strachan A. et al, J. Chem. Phys. 122, 054505 (2005).

4. Dick J.J., J. Appl. Phys. 76, 2726 (1994); 81, 601 (1997).

5. Segall M.D. et al, J. Phys. Cond. Matt. 14, 2717 (2002).

6. Schultz P.A., SeqQuest electronic structure code: http://dft.sandia.gov/Quest/SeqQ Home.htm

7. Olinger B., Halleck P.M., and Cady H.H., J. Chem. Phys. 62, 4480 (1975). 